\title{
Combined Management can Decrease Blood Pressure: A Health-seeking Behaviors Investigation among Hypertensive Patients in Urban Communities in China
}

\author{
Si Wang \\ West China Hospital of Sichuan University \\ Kai Liu \\ West China Hospital of Sichuan University \\ Xin Zhang \\ West China Hospital of Sichuan University \\ Qingtao Meng \\ West China Hospital of Sichuan University \\ Xinran Li \\ West China Hospital of Sichuan University \\ Runyu Ye \\ West China Hospital of Sichuan University \\ Zhipeng Zhang \\ West China Hospital of Sichuan University \\ Xiaoping Chen ( $D$ xiaopingchen11@126.com) \\ West China Hospital of Sichuan University
}

\section{Research Article}

Keywords: health-seeking behaviors, combined management, community health service center, blood pressure, hypertension

Posted Date: February 3rd, 2021

DOI: https://doi.org/10.21203/rs.3.rs-154409/v1

License: (c) (1) This work is licensed under a Creative Commons Attribution 4.0 International License. Read Full License 


\section{Abstract}

Background: Hypertensive patients can freely choose the informal medical facilities such as pharmacies, community health service centers, and cardiology clinics in secondary or tertiary hospitals, as the routine places for medical treatment in China at present. It is not clear the proportion, influencing factors and the effects on blood pressure (BP) of different health-seeking behaviors among hypertensive patients in urban communities. The aim of the study is to investigate the health-seeking behaviors and the effect on $\mathrm{BP}$ of different health-seeking behaviors among hypertensive patients in urban communities in China.

Methods: A cross-sectional survey of hypertension was conducted in urban communities in Chengdu. 437 hypertensive patients seeking medical help regularly were sequentially enrolled to fill out the questionnaire on health-seeking behaviors.

Results: The average age was $67.1 \pm 7.5$ years. Systolic blood pressure (SBP) and diastolic blood pressure (DBP) were $144.2 \pm 17.9 \mathrm{mmHg}$ and $75.4 \pm 10.4 \mathrm{mmHg}$, respectively, and the control rate of BP was $41.0 \%$. Among the hypertensive patients investigated, $5.2 \%$ of which chose the informal medical facilities, $62.8 \%$ chose the community health service center, $21.5 \%$ chose the cardiology clinics in secondary or tertiary hospitals, and $10.5 \%$ chose both community health service center and cardiology clinics as the usual places for medical treatment. There were statistical differences in education levels, proportions of home BP monitoring, establishing chronic disease archives in community, medication compliance and side effects of drugs among the four groups. The SBP was $150.9 \pm 19.8,145.1 \pm 18.0,143.8 \pm 17.5$ and $136.3 \pm 15.1 \mathrm{mmHg}(P=0.007)$ in the four groups respectively and it was significantly lower in the combined management group than in the other three groups. The control rate of BP was $23.8 \%, 39.4 \%$, $43.0 \%$ and $54.8 \%(P=0.100)$, respectively. Compared with patients choosing the informal medical facilities, patients in the combined management group had significant higher $\mathrm{BP}$ control rate $(\mathrm{OR}=3.679$, $P=0.035)$.

Conclusions: Combined management with both community health service center and higher-level hospitals can decrease BP.

\section{Background}

Hypertension is a common cardiovascular risk factor, which can cause damage to the heart, brain and kidney, and can give rise to the occurrence of cardiovascular events such as heart failure, stroke and renal failure. Decreasing blood pressure (BP) to the target can reduce the incidence of cardiovascular events in patients with hypertension greatly $[1,2]$. Hypertension has a high prevalence in China, nearly $23.9 \%$ above 18 years [3], but the control rate is very low, just $5.7 \%$ in a population-based screening study [4] and $38.3 \%$ in urban areas in another cross-sectional study in China [5].

Conducting health management in hypertensive patients relying on the community health service centers is an important part of basic public health service in China. Standardized hypertension management can improve hypertension control rate significantly in community [6-8]. However, due to the different quality 
of basic health services provided by different communities and the different demands of hypertensive patients, not all hypertensive patients take community health service centers as the routine places for medical treatment [9-11]. They can freely choose the informal medical facilities without physicians in attendance, community health service centers, and cardiology clinics in secondary or tertiary hospitals, as the routine places for medical treatment. At present, it is not clear the proportion, influencing factors and the effects on BP of different health-seeking behaviors among hypertensive patients in urban communities in China.

Therefore, we conducted an investigation on the health-seeking behaviors among hypertensive patients in an urban community in Chengdu, a central city in western China, to find out the health-seeking behaviors of hypertensive patients and the influencing factors, and also to reveal the effects on BP of different health-seeking behaviors.

\section{Methods}

\section{Study Design}

We conducted a cross-sectional survey of hypertension for more than 18 years old residents through door-to-door visit and registration in the community activity centers using a random cluster sampling method in 5 randomly selected urban residential communities in Chengdu belonging to the same community health service center in 2011-2012. As shown in Figure 1, a total of 3,775 residents were investigated and 1,438 residents were diagnosed as hypertension. 970 hypertensive patients went voluntarily to the community health service center to receive physical and serological examinations. 437 hypertensive patients seeking medical help regularly were sequentially enrolled to fill out the questionnaire on health-seeking behaviors by trained staffs. At last, 400 questionnaires were filled out in a standardized manner, and the recovery rate was $91.5 \%$. This study was approved by the ethics committee of West China hospital, Sichuan University. Written informed consent was obtained from all participants.

\section{Measurements}

Basic information included name, age, gender, smoking and drinking habits, physical exercise, education level, combined cardiovascular diseases, duration, symptoms and family history of hypertension, home BP monitoring, medication compliance, side effects of drugs, number of antihypertensive drugs and medical insurance reimbursement. Health-seeking behaviors was referred to patients' different choice of medical treatment place. As to physical examinations, the patients were requested to sit for at least 5 minutes, then 3 consecutive BP readings were recorded from the supported right arm at one-minute intervals using a calibrated upper arm electronic sphygmomanometer (OMRON HEM-7200). The BP was calculated as an average value of the three measurements. The other anthropometric data, including height, weight and waist circumference, were measured as what we did before [12]. Serological 
examinations included creatinine, uric acid (UA), glucose, triglyceride (TG), total cholesterol (TC), highdensity lipoprotein cholesterol (HDL-C) and low-density lipoprotein cholesterol (LDL-C). Creatinine was measured by picric acid method, UA, glucose, TG and TC were measured by oxidase method, while HDL-C and LDL-C were measured by the direct method.

\section{Related definitions}

Hypertension was defined as the calculated average systolic blood pressure (SBP) $\geq 140 \mathrm{mmHg}$ and/or diastolic blood pressure (DBP) $\geq 90 \mathrm{mmHg}$ or taking antihypertensive drugs [13]. Diabetes was defined as fasting plasma glucose $\geq 7.0 \mathrm{mmol} / \mathrm{L}$ or taking hypoglycemic drugs [14]. Body mass index (BMI) was calculated as body mass/height ${ }^{2}\left(\mathrm{~kg} / \mathrm{m}^{2}\right)$. Obesity was defined as $\mathrm{BMI} \geq 28 \mathrm{~kg} / \mathrm{m}^{2}$ or waist circumference $\geq 90 \mathrm{~cm}$ for male and $\geq 85 \mathrm{~cm}$ for female [15-16]. Smoking was defined as smoking more than 1 cigarette per day. Alcohol consumption was defined as drinking $\geq 50 \mathrm{ml}$ liquor ( 1 bottle of beer $=100 \mathrm{ml}$ liquor) every time, $\geq 1$ time per week. Regular physical exercise was defined as taking part in physical activities at least 3 times a week and more than 30 minutes every time. BP control was defined as the BP was controlled below $140 / 90 \mathrm{mHg}$. Combined cardiovascular diseases included dyslipidemia, goat, atrial fibrillation, coronary heart disease, heart failure, and stroke. Good medication compliance indicated taking antihypertensive medications more than 9 months a year.

\section{Statistical analysis}

Patients with hypertension were divided into four groups according to the different place they chose for medical treatment. Group 1 was consisted of patients choosing the informal medical facilities without physicians in attendance such as pharmacies as the usual places for medical treatment, group 2 was consisted of patients choosing community health service center as the usual place for medical treatment, group 3 was consisted of patients choosing the cardiology clinics in secondary or tertiary hospitals as the usual places for medical treatment, group 4 named as combined management group was consisted of patients choosing both community health service center and higher-level hospitals as the usual places for medical treatment. Descriptive data was presented as means (SD, standard deviation) and proportions. F test was used for comparison of the characteristics among 4 groups, and LSD test was used for pairwise comparison between groups. Comparison of categorical variables among 4 groups was evaluated with the Pearson $\chi 2$ test. The influence of different health-seeking behaviors on BP control rate was further analyzed using logistic regression analysis by taking group 1 as a reference. SPSS software package (version 18.0; SPSS, Chwicago, IL) was used to conduct statistical analysis. Statistical significance was defined as $\mathrm{P}<0.05$.

\section{Results}

\section{Basic information of the hypertensive patients}


The average age of the hypertensive patients investigated was $67.1 \pm 7.5$ years, and $46.5 \%$ of which were male. The average duration of hypertension was $10.1 \pm 8.7$ years. The mean SBP and DBP were $144.2 \pm 17.9 \mathrm{mmHg}$ and $75.4 \pm 10.4 \mathrm{mmHg}$, respectively, and the total control rate of blood pressure was $41.0 \%$. Among the participants, $18.3 \%$ of which just had cultural degree of primary school and below, $43.2 \%$ had junior high school of education and $38.5 \%$ had high school level of education and above, the proportion of smoking, drinking and exercise was $9.0 \%, 14.3 \%$ and $78.8 \%$, respectively. $12.5 \%$ of the participants were diagnosed as obesity, $46.3 \%$ were diagnosed as abdominal obesity and $22.0 \%$ were diagnosed as diabetes. $71.8 \%$ of which had symptoms of hypertension, $66.3 \%$ performed home BP monitoring, $75.5 \%$ established chronic disease archives in community health service center, $85.0 \%$ had good medication compliance, $32.3 \%$ could adjust antihypertensive drugs by themselves sometimes, $23.5 \%$ had side effects of antihypertensive drugs, and $60.8 \%$ can get part of the reimbursement.

\section{Investigation of patients' health-seeking behaviors and the influencing factors}

Among the hypertensive patients surveyed, 21 patients chose to visit the informal medical facilities, accounting for $5.2 \%, 251$ patients chose to visit the community health service center, accounting for $62.8 \%, 86$ patients chose to see doctors in the cardiology clinics of secondary or tertiary hospitals, accounting for $21.5 \%$, and 42 patients chose both community health service center and higher-level hospitals as the usual places for medical treatment, accounting for $10.5 \%$. As shown in the table 1 , there were no statistical differences in age, gender, waist, weight, BMI, proportion of smoking, drinking and exercise, duration, symptoms and family history of hypertension, serological results and proportion of obesity, diabetes, cardiovascular diseases combined, medical in Otherwise, there were statistical differences in the proportion of education levels, home BP monitoring, establishing chronic disease archives in community, medication compliance and side effects of drugs among the four groups. 
Table 1

The characteristics of the participants in the four groups

\begin{tabular}{|c|c|c|c|c|c|}
\hline Characteristics & Group 1 & Group 2 & Group 3 & Group 4 & $\begin{array}{l}P \\
\text { value }\end{array}$ \\
\hline Number & $21(5.2)$ & $251(62.8)$ & $86(21.5)$ & $42(10.5)$ & \\
\hline Age & $67.9 \pm 7.7$ & $67.0 \pm 7.3$ & $67.9 \pm 7.1$ & $65.3 \pm 9.1$ & 0.283 \\
\hline Gender(male) & $8(38.1)$ & $113(45.0)$ & $47(54.7)$ & $18(42.9)$ & 0.346 \\
\hline Waist, cm & $85.4 \pm 8.2$ & $85.3 \pm 8.6$ & $86.2 \pm 9.0$ & $87.6 \pm 10.4$ & 0.474 \\
\hline Height, cm & $158.5 \pm 6.7$ & $160.3 \pm 7.5$ & $161.4 \pm 8.0$ & $162.5 \pm 8.5$ & 0.135 \\
\hline Weight, kg & $63.0 \pm 10.0$ & $63.0 \pm 9.2$ & $64.1 \pm 10.7$ & $65.3 \pm 10.3$ & 0.496 \\
\hline $\mathrm{BMI}, \mathrm{Kg} / \mathrm{cm}^{2}$ & $25.1 \pm 4.1$ & $24.5 \pm 2.8$ & $24.5 \pm 3.2$ & $24.7 \pm 2.9$ & 0.831 \\
\hline Smoking & $2(9.5)$ & $22(8.8)$ & $9(10.5)$ & $3(7.1)$ & 0.917 \\
\hline Drinking & $3(14.3)$ & $33(13.1)$ & $16(18.6)$ & $5(11.9)$ & 0.622 \\
\hline Exercise & 18(85.7) & 199(79.3) & 61(70.9) & $37(88.1)$ & 0.126 \\
\hline \multicolumn{6}{|l|}{ Education levels } \\
\hline Primary school and below & $8(38.1)$ & $51(20.3)$ & $7(8.1)$ & $7(16.7)$ & \multirow[t]{3}{*}{0.028} \\
\hline Junior high school & $9(42.9)$ & $107(42.6)$ & $40(46.5)$ & $17(40.5)$ & \\
\hline High school and above & $4(19.0)$ & $93(37.1)$ & $39(45.3)$ & 18(42.9) & \\
\hline Obesity & $5(23.8)$ & $30(12.0)$ & $9(10.5)$ & $6(14.3)$ & 0.401 \\
\hline Abdominal obesity & $8(38.1)$ & $116(46.2)$ & $37(43.0)$ & $24(57.1)$ & 0.408 \\
\hline Diabetes & $4(19.0)$ & $51(20.3)$ & $23(26.7)$ & $10(23.8)$ & 0.64 \\
\hline \multicolumn{6}{|c|}{ Cardiovascular diseases combined } \\
\hline 0 & $11(52.4)$ & $130(51.8)$ & $34(39.5)$ & $18(42.9)$ & \multirow[t]{3}{*}{0.54} \\
\hline 1 & $6(28.6)$ & $75(29.9)$ & $34(39.5)$ & 16(38.1) & \\
\hline$\geq 2$ & $4(19.0)$ & $46(18.3)$ & 18(20.9) & $8(19.0)$ & \\
\hline Duration of hypertension & $10.1 \pm 7.1$ & $9.6 \pm 8.8$ & $11.2 \pm 8.7$ & $10.5 \pm 8.7$ & 0.544 \\
\hline Symptoms of hypertension & $12(57.1)$ & 185(73.7) & $57(66.3)$ & $33(78.6)$ & 0.179 \\
\hline Family history of hypertension & $8(38.1)$ & $99(39.4)$ & $31(36.0)$ & $21(50.0)$ & 0.504 \\
\hline Home BP monitoring & 16(76.2) & $149(59.4)$ & $67(77.9)$ & $33(78.6)$ & $<0.001$ \\
\hline Chronic disease archives & $11(52.4)$ & $202(80.5)$ & $53(61.6)$ & $36(85.7)$ & $<0.001$ \\
\hline
\end{tabular}




\begin{tabular}{|c|c|c|c|c|c|}
\hline Medication compliance & $17(81.0)$ & $207(82.1)$ & $75(87.2)$ & $41(97.6)$ & 0.033 \\
\hline Adjusting drugs & $7(33.3)$ & 83(33.1) & $24(27.9)$ & 15(35.7) & 0.788 \\
\hline Side effects of drugs & $4(19.0)$ & $61(24.3)$ & $11(12.8)$ & $14(43.8)$ & 0.005 \\
\hline \multicolumn{6}{|l|}{ Anti-hypertensive drugs } \\
\hline 1 drug & $12(57.1)$ & $151(60.2)$ & $46(54.1)$ & $24(57.1)$ & \multirow[t]{3}{*}{0.468} \\
\hline$\geq 2$ drugs & $5(23.8)$ & $69(27.5)$ & $33(38.8)$ & 14(33.3) & \\
\hline Chinese patent drugs & $4(19.0)$ & $31(12.4)$ & $7(8.1)$ & $4(9.5)$ & \\
\hline $\begin{array}{l}\text { Medical insurance } \\
\text { reimbursement }\end{array}$ & $9(42.9)$ & $152(60.6)$ & $55(64.0)$ & $27(64.3)$ & 0.338 \\
\hline Creatinine, $\mathrm{mmol} / \mathrm{L}$ & $94.9 \pm 18.7$ & $91.9 \pm 15.2$ & $96.2 \pm 16.9$ & $92.4 \pm 14.4$ & 0.323 \\
\hline $\mathrm{UA}, \mu \mathrm{mmol} / \mathrm{L}$ & $363.7 \pm 111.0$ & $363.1 \pm 94.8$ & $395.4 \pm 95.2$ & $340.6 \pm 91.8$ & 0.066 \\
\hline Glucose, $\mathrm{mmol} / \mathrm{L}$ & $6.44 \pm 2.41$ & $5.94 \pm 1.56$ & $6.39 \pm 1.67$ & $5.97 \pm 1.25$ & 0.227 \\
\hline $\mathrm{TG}, \mathrm{mmol} / \mathrm{L}$ & $1.70 \pm 0.69$ & $1.60 \pm 0.72$ & $1.77 \pm 1.01$ & $1.60 \pm 0.93$ & 0.543 \\
\hline $\mathrm{TC}, \mathrm{mmol} / \mathrm{L}$ & $5.28 \pm 0.87$ & $5.20 \pm 3.03$ & $4.85 \pm 1.04$ & $4.96 \pm 1.08$ & 0.783 \\
\hline $\mathrm{HDL}-\mathrm{C}, \mathrm{mmol} / \mathrm{L}$ & $1.65 \pm 0.51$ & $1.48 \pm 0.33$ & $1.41 \pm 0.34$ & $1.52 \pm 0.33$ & 0.076 \\
\hline LDL-C, mmol/L & $2.66 \pm 0.92$ & $2.50 \pm 0.71$ & $2.56 \pm 0.72$ & $2.42 \pm 0.79$ & 0.823 \\
\hline \multicolumn{6}{|c|}{ Data are presented as means \pm SD or number (percentage). } \\
\hline \multicolumn{6}{|c|}{$\begin{array}{l}\text { BMI: body mass index; UA: uric acid; TG: triglyceride; TC: total cholesterol; HDL-C: high density } \\
\text { lipoprotein-cholesterol; LDL-C: low density lipoprotein-cholesterol. }\end{array}$} \\
\hline
\end{tabular}

\section{Effects of different health-seeking behaviors on BP level and control rate}

As shown in Figure 2-3, the SBP in the four groups were 150.9 $\pm 19.8,145.1 \pm 18.0,143.8 \pm 17.5$ and $136.3 \pm 15.1 \mathrm{mmHg}$, respectively, the difference was statistically significant $(P=0.007)$. The SBP in the combined management group was significantly lower than in the other three groups through pairwise comparison. The DBP in the four groups were $79.0 \pm 8.5,75.9 \pm 10.5,74.3 \pm 10.7$ and $72.6 \pm 9.7 \mathrm{mmHg}$, respectively, the difference was not statistically significant $(P=0.066)$. As shown in Figure 4 , the control rate of $B P$ in the four groups were $23.8 \%, 39.4 \%, 43.0 \%$ and $54.8 \%(P=0.100)$, respectively. Table 2 shows the results of logistic regression analysis for the relationship of health-seeking behaviors and BP control in different models. Compared with patients in group 1 who chose the informal medical facilities as the usual places for medical treatment, patients in the combined management group had significant higher 
BP control rate $(\mathrm{OR}=3.874, \mathrm{P}=0.024)$ in unadjusted logistic analysis model. After adjusting for age, gender, education level, duration of hypertension and medication compliance, patients in the combined management group still had significant higher $B P$ control rate $(O R=3.679, P=0.035)$ than in group 1 .

Table 2

Logistic analysis for the influence of health-seeking behaviors on BP control

\begin{tabular}{|lllllll|}
\hline \multicolumn{5}{|c|}{ Unadjusted } & \multicolumn{5}{c|}{ Adjusted } \\
\cline { 2 - 6 } & OR & $95 \% \mathrm{Cl}$ & P value & OR & $95 \% \mathrm{Cl}$ & $95 \% \mathrm{Cl}$ \\
\hline Reference=Group 1 & & & & & \\
\hline Group 2 & 2.084 & $0.74-5.871$ & 0.165 & 1.998 & $0.69-5.79$ & 0.202 \\
\hline Group 3 & 2.416 & $0.811-7.195$ & 0.113 & 2.519 & $0.814-7.792$ & 0.109 \\
\hline Group 4 & 3.874 & $1.198-12.527$ & 0.024 & 3.679 & $1.097-12.336$ & 0.035 \\
\hline $\begin{array}{l}\text { OR, odds ratio; Cl, confidence interval. The unadjusted model was conducted by univariate regression } \\
\text { analysis; the adjusted model was conducted adjusting for age, gender, education level, duration of } \\
\text { hypertension and medication compliance. }\end{array}$ \\
\hline \multicolumn{7}{|l}{} \\
\hline
\end{tabular}

\section{Discussion}

At present, few studies have reported the medical institutions choice of hypertensive patients. Dafeng et al. investigated the health-seeking behaviors of 510 patients with hypertension or diabetes in Wuhan in China, and the results showed that $75.35 \%$ of patients chose higher-level hospitals for medical treatment [17]. Another community-based study conducted in Guangdong province in China including 154,504 subjects who had a usual source of healthcare demonstrated that $65.3 \%$ of patients used outpatient services at primary care regularly for tackling chronic diseases [18]. However, the combined healthseeking behaviors has not been reported in the two studies and it was somewhat different from the result of our study. The population in our study was all from the residents in the central urban area of Chengdu, a central city in western China, with good economic development and community health service construction. We can see that the patients' health-seeking behaviors are completely different due to unbalanced economic development and different maturity levels of community health service centers in China. Further researches need to be done in different regions at different levels of economic development. The results of our study can only represent the health-seeking behaviors of hypertensive patients in the central urban areas.

Health-seeking behaviors is defined as any activity undertaken by individuals who perceive themselves to have a health problem or to be ill for the purpose of finding an appropriate remedy [19]. The desired health-seeking behaviors is responding to an illness by seeking help from a trained allopathic doctor in a recognized health care center [20]. Musinguzi GS et al. found that factors influencing health-seeking behaviors were related to health systems and the patient socioeconomic and structural environment [19]. 
The main system issues were related to availability and attitudes of staff and shortage of supplies and medicines [21]. The patient factors were related to awareness, perceived severity, perceived effectiveness of therapy, adverse effects, and perceived fears of lifelong dependence on medicines [22]. However, the influencing factors of different health-seeking behaviors are not clear at present. Neither of the two studies we mentioned before have conducted further researches on the factors influencing the different health-seeking behaviors $[17,18]$. Our studies demonstrated that education levels and side effects of drugs had impacts on the patients' health-seeking behaviors. The patients choosing higher-level hospitals as the usual places for medical treatment had the largest proportion of middle and advanced education level, and the patients choosing the informal medical facilities as the usual places for medical treatment had the largest proportion of low education level. This is just the same as what Fjaer EL et al. found, that higher socioeconomic position groups are more likely to use health care specialists, compared with lower socioeconomic position groups, and in the context of health care specialist use, education and occupation appear to be particularly important factors [23]. However, the proportion of patients with middle and advanced education was not the highest in the combined health-seeking group. It indicated that education level is not an important factor influencing the choice of combined health-seeking behaviors. Otherwise, the incidence of adverse effects was the highest in the combined group. Although studies have shown that adverse effect is an influential factor for drug adherence [24-26]. However, side effects of drugs may also prompt patients to seek medical treatment from community health service center to higher-level hospitals in our study,so as to receive combined therapy from community health service center and higher-level hospitals. It may also because patients in the combined management group pay more attention to the drug response.

The community-based epidemiological survey of hypertension showed that the overall control rate of hypertension was poor, as we mentioned before, just $5.7 \%$ in a population-based screening study [4] and $38.3 \%$ in urban areas, $17.5 \%$ in rural areas in another cross-sectional study in China [5]. In the survey conducted in the outpatient, including 5,206 hypertensive patients from 46 hypertension outpatient clinics in 22 provinces, autonomous regions, and municipalities of China, the control rate of hypertension was $44.3 \%$, higher than the control rate obtain in the community [27]. Our study showed that the BP control rate of hypertensive patients who chose higher-level hospitals as the usual places for medical treatment was $43.0 \%$, which was similar to the control rate in previous studies [27]. The control rate of hypertensive patients who chose community health service center as the usual place for medical treatment was $39.4 \%$, which was close to the control rate in group choosing higher-level hospitals. In addition, patients with hypertension who chose the informal medical facilities as the usual places for medical treatment had the lowest BP control rate, which was only $23.8 \%$. The control rate was the highest in the combined management group, reached $54.8 \%$, higher than that in other studies $[4,5,27]$, and the average SBP was $136.3 / 72.6 \mathrm{mmHg}$ in this group. Why was the level of BP the lowest in the combined management group? As we can see, there was no significant difference in the number of antihypertensive drugs among the four groups. And the proportion of combined medication use was relatively higher in the group choosing the higher-level hospitals for medical treatment, but the BP control rate was lower than that in the group with combined health-seeking behaviors. Therefore, it was not the number of drugs that makes 
the difference in BP. The rationality of prescription and the timeliness of prescription adjustment may play a more important role. As we all know, the drug regimens cannot be more precisely adjusted in patients treated in community health service center when the BP is difficult to control, and the medical problems cannot be solved in time sometimes in patients who simply choose the higher-level hospitals for medical treatment. In the combined management group, patients can not only receive medical services from the community health service center conveniently, but also enjoy better medical services from higher-level hospitals. They would like to visit higher-level hospitals if their BP can't be controlled to normal in the community health service center and they had the highest medication compliance.

However, the loose collaborative management in our study have not significantly improved the BP control rate of hypertensive patients. Yi Q, et al. randomly chose 218 primary hypertensive patients from a community health service center to investigate the effect of the integration pattern of hospital-community on the grade-based management for hypertension in elders. After 6 months of intervention, the control rate in the patients elevated from 22.9-88.1\% [28], higher than the BP control rate of the combined management group in our study. But there was no control group, and the evaluation of health economics and the concrete working mode should be further discussed.

Our study has some limitations. First, the sample size was not large enough to get statistical differences in BP control rate among the 4 groups. But there were statistically significant differences in BP levels among the 4 groups. Although we conducted the survey using a random cluster sampling method, two thirds of the hypertensive patients went voluntarily to the community health service center to receive physical and serological examinations. Those seeking medical help regularly were sequentially enrolled to fill out the questionnaire on health-seeking behaviors. Therefore, there may be some selection bias in our study, which may have an impact on the composition ratio of health-seeking behaviors, but it can also reflect the influence of different health-seeking behaviors on BP. The third, it was a cross-sectional community sampling survey to evaluate the influence of different health-seeking behaviors on BP control rate. As the control rate of $\mathrm{BP}$ was also affected by many other factors which could not be corrected one by one, it could only provide a reference for further studies in the future. It is important to further study the effect of hospital-community combined hypertension management on the improvement of the control rate of hypertension and further explore the reasonable combined management mode. In addition, the survey was conducted in the central urban area of Chengdu, and the construction of the community chronic disease management system is relatively complete, it can only represent the characteristics of such type communities. The appropriate management pattern of hypertension in different regions of China still needs to be further studied.

\section{Conclusions}

In conclusion, the combined management of hypertensive patients taking advantage of the superior medical resources in the higher-level hospitals, to carry out disease evaluation and make reasonable drug treatment plans, and then transferring the patients to the community health service center for follow-up management may be an effective way to improve the control rate of hypertension. But how to operate the combined management efficiently needs further study. We have conducted a study to evaluate the 
clinical efficacy and health economics effect of an internet-based patient-primary care physiciancardiologist integrated management model of hypertension in China [29]. We are looking forward to the results.

\section{Abbreviations}

BP: Blood pressure; SBP: Systolic blood pressure; DBP: Diastolic blood pressure; UA: Uric acid; TG: Triglyceride; TC: Total cholesterol; HDL-C: High-density lipoprotein cholesterol; LDL-C: Low-density lipoprotein cholesterol; BMI: Body mass index; OR: Odds ratio; $\mathrm{Cl}$, Confidence interval; SD: Standard deviation.

\section{Declarations}

\section{Ethics approval and consent to participate}

This study was approved by the ethics committee of West China hospital, Sichuan University. Written informed consent was obtained from all participants.

\section{Consent to publish}

Not Applicable.

\section{Availability of data and materials}

The data can be obtained from the corresponding author under reasonable request.

\section{Competing interests}

The authors declare that they have no conflict of interest.

\section{Funding}

This study was supported by the project of the Science and Technology Department in Sichuan Province (No. 2012SZ0131).

\section{Authors' contributions}

SW, $\mathrm{KL}$ and $\mathrm{XZ}$ have participated in the design of the study, performed the statistical analyses and drafted the manuscript. QTM, XRL, RYY and ZPZ have participated in the analysis and interpretation of 
data and helped to draft and review the manuscript. XPC helped in acquisition of data and final draft preparation. All of the authors have read and approved the final manuscript.

\section{Acknowledgements}

We thank all patients who took part in the studies and the staffs in Jinyang Community Health Service Center, Wuhou District, Chengdu.

\section{Author details}

Department of Cardiovascular Medicine, West China Hospital, Sichuan University, Chengdu 610041, China

\section{References}

1. Williams B, Mancia G, Spiering W, Agabiti Rosei E, Azizi M, Burnier M, et al. 2018 ESC/ESH Guidelines for the management of arterial hypertension: The Task Force for the management of arterial hypertension of the European Society of Cardiology and the European Society of Hypertension. $J$ Hypertens. 2018;36:1953-2041.

2. Cifu AS, Davis AM. Prevention, Detection, Evaluation, and Management of High Blood Pressure in Adults. JAMA. 2017;318:2132-34.

3. Liu X, Gu W, Li Z, Lei H, Li G, Huang W. Hypertension prevalence, awareness, treatment, control, and associated factors in Southwest China: an update. J Hypertens. 2017;35:637-44.

4. Lu J, Lu Y, Wang X, Li X, Linderman GC, Wu C, Cheng X, et al. Prevalence, awareness, treatment, and control of hypertension in China: data from 1.7 million adults in a population-based screening study (China PEACE Million Persons Project). Lancet. 2017;390: 2549-58.

5. Zhao L, Sun W, Wang J, Wu J, Zhang Y, Liu Y, et al. Differences in the treatment and control of hypertension in urban and rural residents of the northeastern region of the People's Republic of China: a cross-sectional study. Clin Exp Hypertens. 2019;41:366-72.

6. Zeng XY, Zhang M, Li YC, Huang ZJ, Wang LM. Study on effects of community-based management of hypertension patients aged $\geq 35$ years and influencing factors in urban and rural areas of China, 2010. Zhonghua Liu Xing Bing Xue Za Zhi. 2016;37:612-7.

7. Wang Z, Hao G, Wang X, Wang W, Chen W, Zhu M. Short-term hypertension management in community is associated with long-term risk of stroke and total death in China: A community controlled trial. Medicine (Baltimore). 2016;95:e5245.

8. Patel P, Ordunez P, DiPette D, Escobar MC, Hassell T, Wyss F, et al. Improved Blood Pressure Control to Reduce Cardiovascular Disease Morbidity and Mortality: The Standardized Hypertension Treatment and Prevention Project. J Clin Hypertens (Greenwich). 2016;18:1284-94. 
9. Li T, Lei T, Xie Z, Zhang T. Determinants of basic public health services provision by village doctors in China: using non-communicable diseases management as an example. BMC Health Serv Res. 2016;16:42.

10. Zhao Y, Cui S, Yang J, Wang W, Guo A, Liu Y, et al. Basic public health services delivered in an urban community: a qualitative study. Public Health. 2011;125:37-45.

11. Wang HH, Wang JJ, Wong SY, Wong MC, Li FJ, Wang PX, et al. Epidemiology of multimorbidity in China and implications for the healthcare system: cross-sectional survey among 162,464 community household residents in southern China. BMC Med. 2014;12:188.

12. Wang S, Liu K, Zhang X, Meng Q, Wang Y, Wan S, et al. Elevated resting heart rate predisposes metabolic syndrome in women rather than in men: a 15-year prospective study. BMC Cardiovasc Disord. 2015;15:110.

13. Mancia G, Fagard R, Narkiewicz K, Redon J, Zanchetti A, Böhm M, et al. 2013 ESH/ESC Practice Guidelines for the Management of Arterial Hypertension. Blood Press. 2014;23:3-16.

14. American Diabetes Association. Standards of medical care in diabetes-2013. Diabetes Care. 2013;36 Suppl 1:11-66.

15. Chinese Society of Endocrinology. The expert consensus on Chinese adult obesity prevention and control. Chin J Endocrinol Metab. 2011;27:711-7.

16. Bao Y, Lu J, Wang C, Yang M, Li H, Zhang X, et al. Optimal waist circumference cutoffs for abdominal obesity in Chinese. Atherosclerosis. 2008;201:378-84.

17. Feng D, Serrano R, Ye T, Tang S, Duan L, Xu Y, et al. What Contributes to the Regularity of Patients with Hypertension or Diabetes Seeking Health Services? A Pilot Follow-Up, Observational Study in Two Sites in Hubei Province, China. Int J Environ Res Public Health. 2016;13:E1268.

18. Wang HH, Wang JJ, Wong SY, Wong MC, Li FJ, Wang PX, et al. Epidemiology of multimorbidity in China and implications for the healthcare system: cross-sectional survey among 162,464 community household residents in southern China. BMC Med. 2014;12:188.

19. Musinguzi G, Anthierens S, Nuwaha F, Van Geertruyden JP, Wanyenze RK, Bastiaens H. Factors Influencing Compliance and Health Seeking Behaviour for Hypertension in Mukono and Buikwe in Uganda: A Qualitative Study. Int J Hypertens. 2018; doi:10.1155/2018/8307591.

20. Conner MNP. The theory of planned behaviour and health behaviours. In: Conner M, Norman P (eds). Predicting Health Behaviours: Research and Practice with Social Cognition Models. (Open University Press, Buckingham, 1996) pp 121-62.

21. Kayima J, Wanyenze RK, Katamba A, Leontsini E, Nuwaha F. Hypertension awareness, treatment and control in Africa: a systematic review. BMC Cardiovasc Disord. 2013;13:54.

22. Bourne PA, Morris C, Charles CA, Eldemire-Shearer D, Kerr-Campbell MD, Crawford TV. Health literacy and health seeking behavior among older men in a middle-income nation. Patient Relat Outcome Meas. 2010;1:39-49.

23. Fjær EL, Balaj M, Stornes P, Todd A, McNamara CL, Eikemo TA. Exploring the differences in general practitioner and health care specialist utilization according to education, occupation, income and 
social networks across Europe: findings from the European social survey (2014) special module on the social determinants of health. Eur J Public Health. 2017;27 Suppl 1:73-81.

24. Erdine S. Compliance with the treatment of hypertension: the potential of combination therapy. J Clin Hypertens (Greenwich). 2010;12:40-6.

25. Lobo MD, Sobotka PA, Pathak A. Interventional procedures and future drug therapy for hypertension. Eur Heart J. 2017;38:1101-11.

26. Tedla YG, Bautista LE. Drug Side Effect Symptoms and Adherence to Antihypertensive Medication. Am J Hypertens. 2016;29:772-9.

27. Liu J, Wang W, Liu J, Qi Y, Sun JY, Zhao D. Clustering of cardiovascular risk factors and hypertension control status among hypertensive patients in the outpatient setting. Zhonghua Xin Xue Guan Bing Za Zhi. 2013;41:1050-4.

28. Yi Q, Yang Y, An R, Huang H, Ouyang M, Dai J. Effect of the integration pattern of hospital-community on the grade-based management for hypertension in elders. Zhong Nan Da Xue Xue Bao Yi Xue Ban. 2015;40:1258-63.

29. Runyu Ye, Rufeng Shi, Kai Liu, Xin Zhang, Si Wang, Hang Liao, et al. Internet-based patient- primary care physician-cardiologist integrated management model of hypertension in China: study protocol for a multicentre randomised controlled trial.BMJ Open. 2020; doi: 10.1136/bmjopen-2020-039447.

\section{Figures}

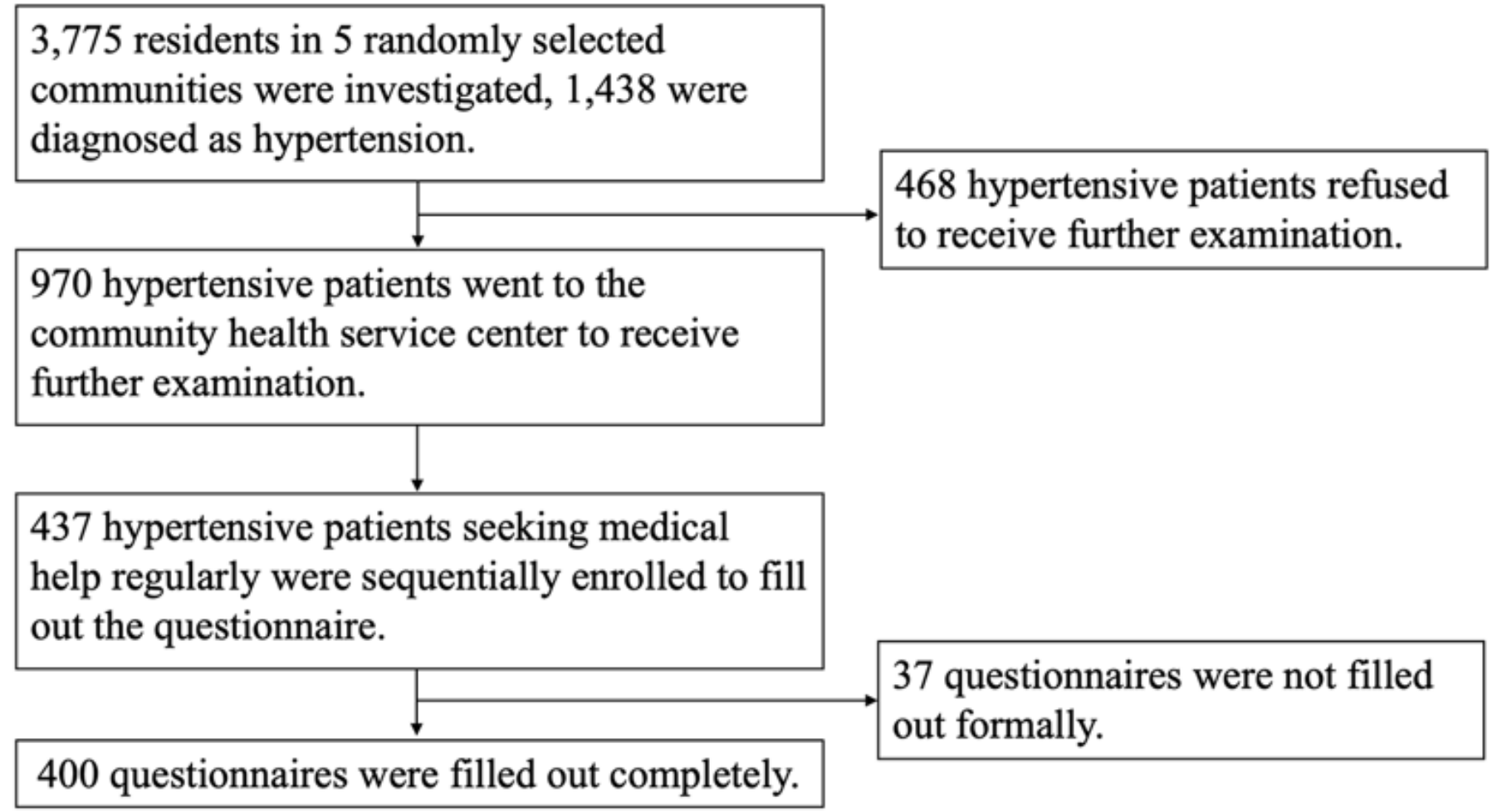

Figure 1 
Recruitment of Survey Participants

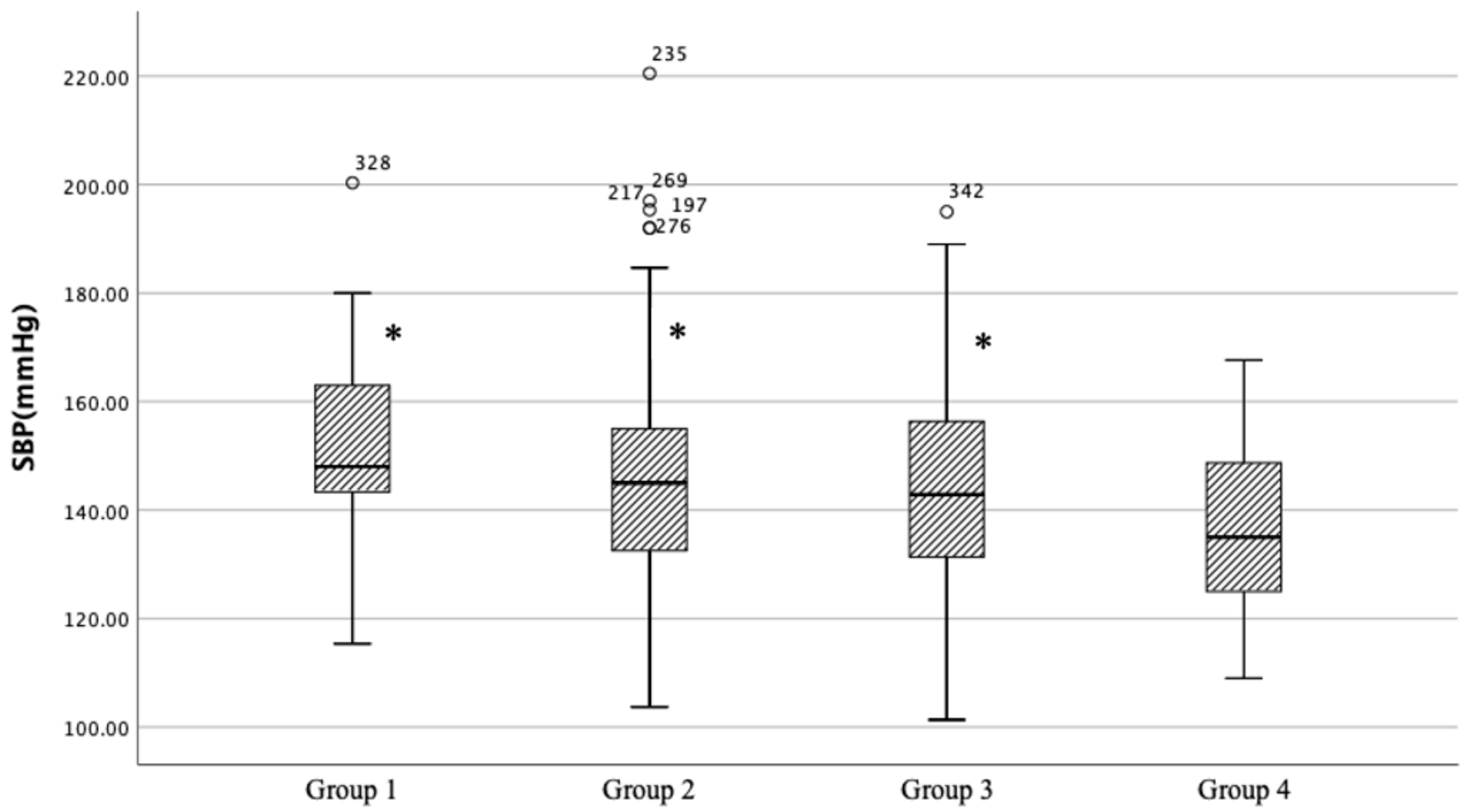

Figure 2

The SBPs in the four groups SBP: systolic blood pressure ${ }^{*}<0.05$ when compared with group 4 through pairwise comparison.

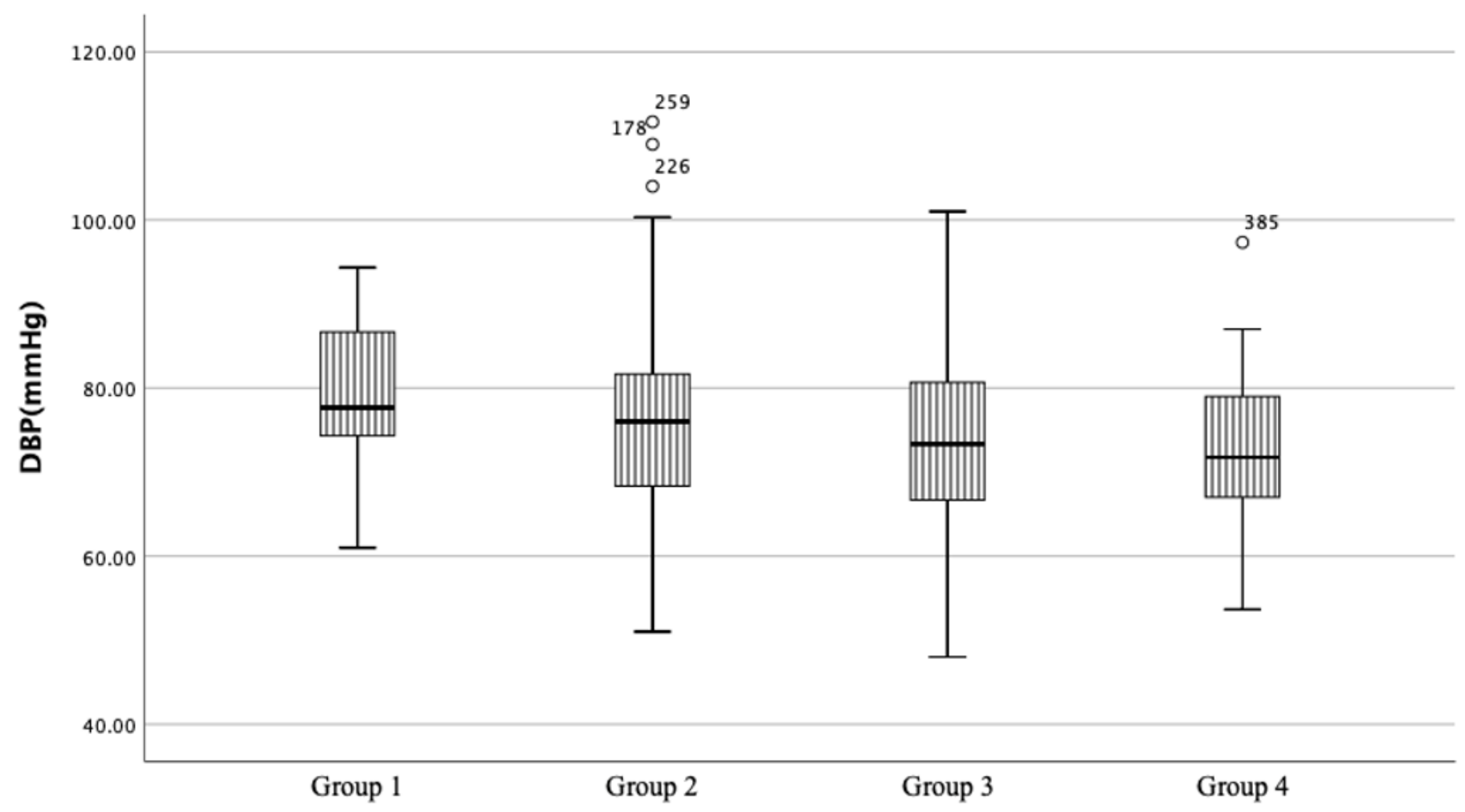


Figure 3

The DBPs in the four groups DBP: diastolic blood pressure

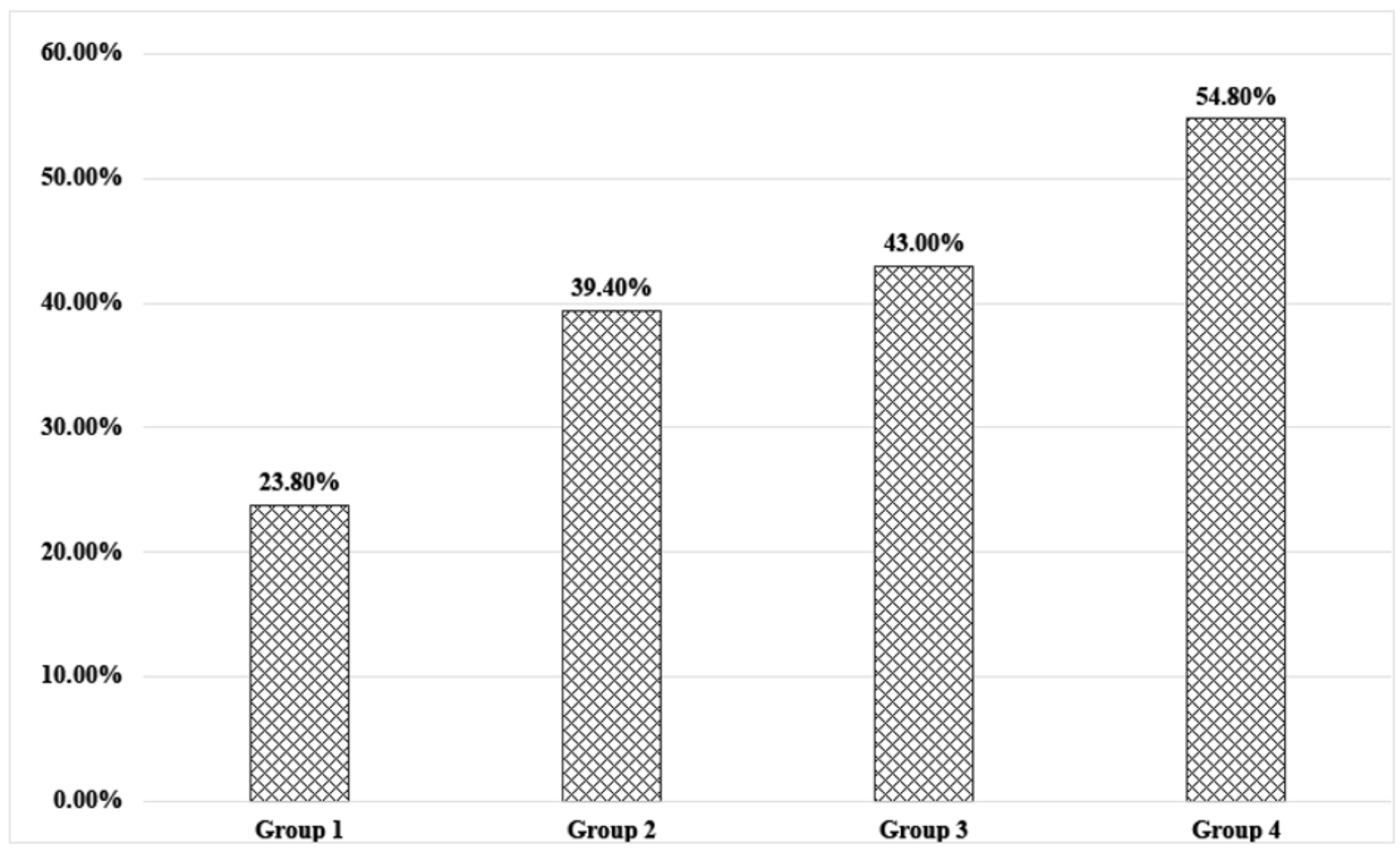

Figure 4

The control rates of BP in the four groups BP: blood pressure 